\title{
Characterisation and 3D Visualisation of Biomaterials and Tissues using Focused Ion Beam (E)SEM
}

\author{
D.J. Stokes, ${ }^{*}$ J.R. Tong, ${ }^{* *}$ J. Juhasz, ${ }^{* * *}$ P.A. Midgley ${ }^{* *} \&$ S.M. Best ${ }^{* * *}$ \\ * c/o Biological \& Soft Systems, Cavendish Laboratory, University of Cambridge, Dept. of Physics, \\ Madingley Road, Cambridge, CB3 OHE. UK \\ ${ }^{* *}$ Electron Microscopy Group, Dept. of Materials Science and Metallurgy, University of Cambridge, \\ Pembroke Street, Cambridge, UK \\ *** Cambridge Centre for Medical Materials, Dept. of Materials Science and Metallurgy, University \\ of Cambridge, Pembroke Street, Cambridge, UK
}

The combination of low vacuum/ESEM and focused ion beam technology represents a powerful new tool for the study of electrically insulating and biological specimens. Low vacuum SEM is known for charge-controlled imaging of uncoated non-conductive materials, while ESEM goes a stage further and allows observation of moist or liquid specimens. Focused ion beam (FIB) instruments have recently attracted a surge of interest as their scope has expanded from the semiconductor industry into the mainstream of materials and life sciences.

Biomaterials such as ceramics, glasses and polymers, along with tissue such as bone represent a class of insulating materials that lend themselves very well to low vacuum imaging, as has previously been demonstrated [1]. However, one challenge is to maintain electrically stable conditions in the high vacuum required for FIB milling. Further details can be found elsewhere [2].

Meanwhile, FIB technology allows us to develop a deeper understanding of the internal microstructure of specimens. For example, by appropriate selection, we can cross-section through interfaces or create a series of 'slices' at specific intervals in the material. Software can then be used to volume-render 3-dimensional information from the 2-dimensional slices, enabling us to immediately see the spatial relationships between heterogeneous microstructural components.

Figure 1 depicts a cubic deposit on the surface of hydroxyapatite (HA) after treatment with simulated body fluid and human serum proteins. Using FIB-milled cross-sections, such as shown in Figure 2, and performing EDS microanalysis, we were able to observe the bulk nature of these deposits and, crucially, infer the presence of calcium and phosphorous.

Meanwhile, the structure and composition of HA may have a significant influence on bone apposition, and so it is of great interest to study how and where bone apposition occurs. Figure 3 is a 3-dimensional reconstruction (Amira software) of dense, cortical bone, showing osteocyte lacunae and associated canaliculi. This visualisation gives far more information than the 2-D images alone.

\section{References}

[1] D.J Stokes et al, Mat. Soc. Res. Symp. Proc. 711 (2002) 113 -118

[2] D.J. Stokes, F. Morrissey, W. R. Knowles \& A.M. Donald, elsewhere in these proceedings

[3] Pfizer, EPSRC and FEI Company are thanked for financial support, and the latter for the loan of a Quanta 3D. The loan of an Oxford Instruments Inca EDS system is also gratefully acknowledged 

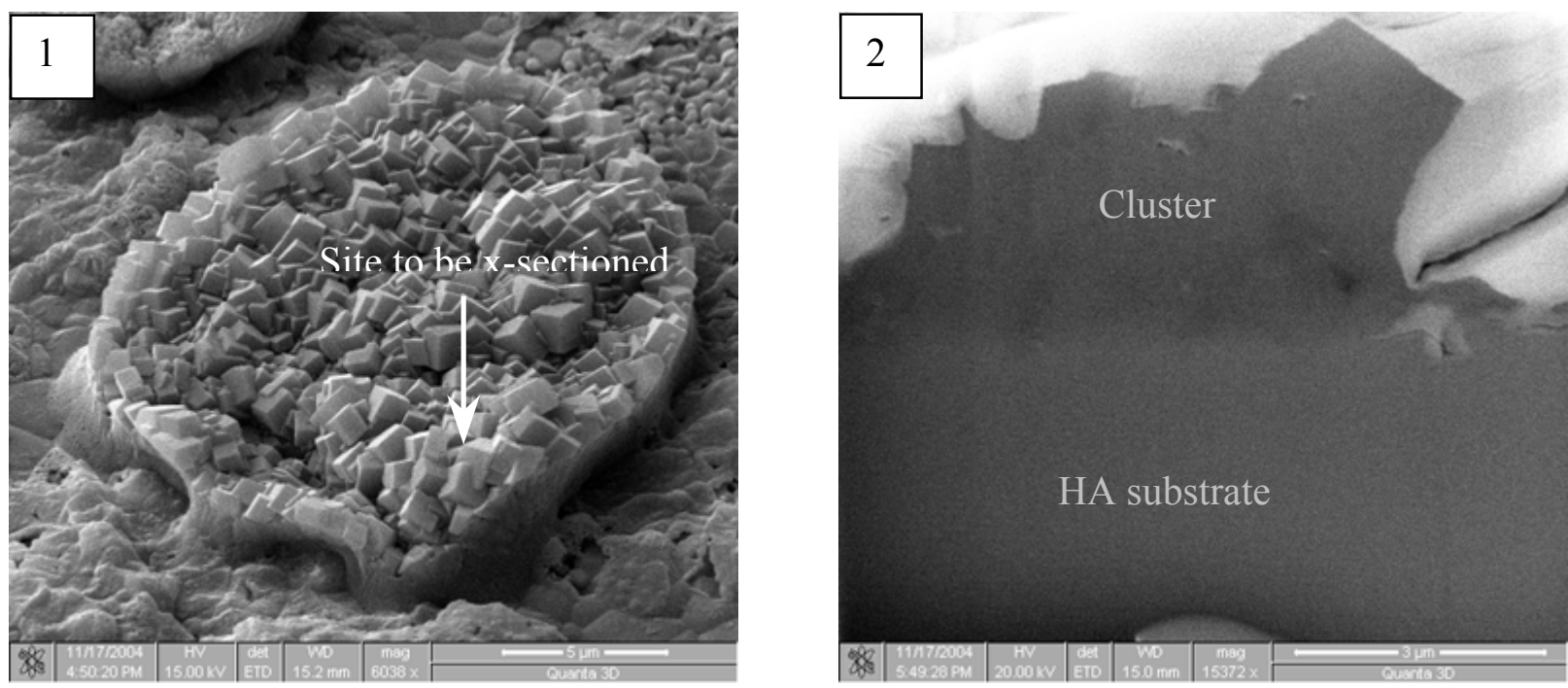

Figure 1. An unusual cubic cluster formed on a hydroxyapatite substrate after immersion in SBF and human serum proteins. Arrow shows region to be FIB milled, as shown in Figure 2.

Figure 2. FIB section through cluster and substrate. EDS analysis confirms that the chemistry of the cluster is highly unusual.

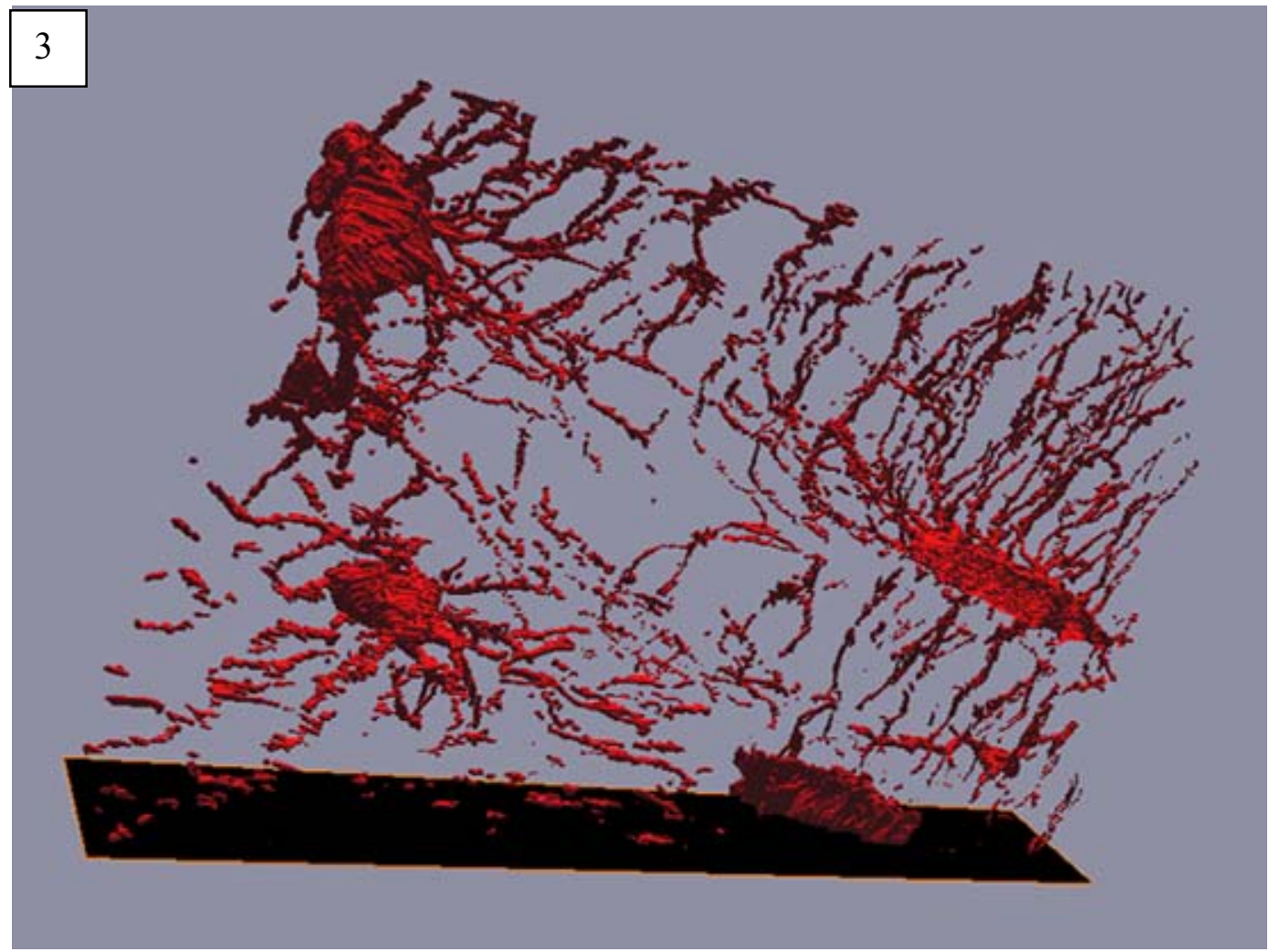

Figure 3. Amira 3-D reconstruction of osteocytes and canaliculi in bone. Note how the complex interactions are revealed when viewed in this way. Horizontal field width $\sim 45$ microns. 